\title{
Long-Term Feature Banks for Detailed Video Understanding
}

\author{
Chao-Yuan $\mathrm{Wu}^{1,2}$ \\ Kaiming $\mathrm{He}^{2}$ \\ Christoph Feichtenhofer ${ }^{2}$ \\ Haoqi Fan $^{2}$ \\ Philipp Krähenbühl ${ }^{1}$ \\ Ross Girshick ${ }^{2}$ \\ ${ }^{1}$ The University of Texas at Austin \\ ${ }^{2}$ Facebook AI Research (FAIR)
}

\begin{abstract}
To understand the world, we humans constantly need to relate the present to the past, and put events in context. In this paper, we enable existing video models to do the same. We propose a long-term feature bank-supportive information extracted over the entire span of a video-to augment state-of-the-art video models that otherwise would only view short clips of 2-5 seconds. Our experiments demonstrate that augmenting $3 D$ convolutional networks with a long-term feature bank yields state-of-the-art results on three challenging video datasets: AVA, EPIC-Kitchens, and Charades. Code is available online.
\end{abstract}

\section{Introduction}

What is required to understand a movie? Many aspects of human intelligence, for sure, but memory is particularly important. As a film unfolds there's a constant need to relate whatever is happening in the present to what happened in the past. Without the ability to use the past to understand the present, we, as human observers, would not understand what we are watching.

In this paper, we propose the idea of a long-term feature bank that stores a rich, time-indexed representation of the entire movie. Intuitively, the long-term feature bank stores features that encode information about past and (if available) future scenes, objects, and actions. This information provides a supportive context that allows a video model, such as a 3D convolutional network, to better infer what is happening in the present (see Fig. 1, 2, and 5).

We expect the long-term feature bank to improve stateof-the-art video models because most make predictions based only on information from a short video clip, typically $2-5$ seconds $[5,33,46-48,51,52,56]$. The reason for this short-term view is simple: benchmark advances have resulted from training end-to-end networks that use some form of 3D convolution, and these 3D convolutions require dense sampling in time to work effectively. Therefore, to fit in GPU memory the video inputs must be short.

\footnotetext{
${ }^{1}$ https://github.com/facebookresearch/ video-long-term-feature-banks
}

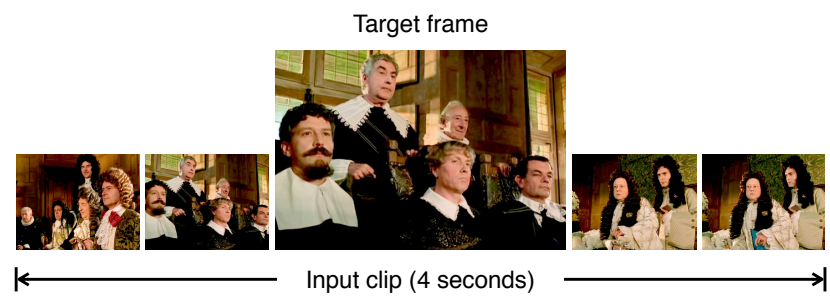

Figure 1. What are these people doing? Current 3D CNN video models operate on short clips spanning only $\sim 4$ seconds. Without observing longer-term context, recognition is difficult. (Video from the AVA dataset [14]; see next page for the answer.)

The long-term feature bank is inspired by works that leverage long-range temporal information by using precomputed visual features [25, 31, 45, 57]. However, these approaches extract features from isolated frames using an ImageNet pre-trained network, and then use the features as input into a trained pooling or recurrent network. Thus the same features represent both the present and the long-term context. In contrast, we propose to decouple the two: the long-term feature bank is an auxiliary component that augments a standard video model, such as a state-of-the-art 3D CNN. This design enables the long-term feature bank to store flexible supporting information, such as object detection features, that differ from what the 3D CNN computes.

Integrating the long-term feature bank with $3 \mathrm{D} \mathrm{CNNs}$ is straightforward. We show that a variety of mechanisms are possible, including an attentional mechanism that relates information about the present (from the 3D CNN) to longrange information stored in the long-term feature bank. We illustrate its application to different tasks with different output requirements: we show results on datasets that require object-level as well as frame- or video-level predictions.

Finally, we conduct extensive experiments demonstrating that augmenting 3D CNNs with a long-term feature bank yields state-of-the-art results on three challenging video datasets: AVA spatio-temporal action localization [14], EPIC-Kitchens verb, noun, and action classification [6], and Charades video classification [38]. Our ablation study establishes that the improvements on these tasks arise from the integration of long-term information. 


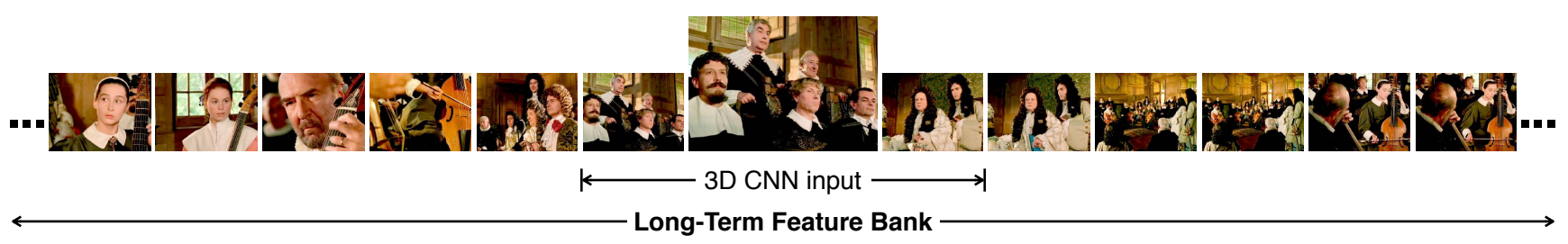

Figure 2. The action becomes clear when relating the target frame to the long-range context. Our long-term feature bank provides longterm supportive information that enables video models to better understand the present. (AVA ground-truth label: 'listening to music'.)

\section{Related Work}

Deep networks are the dominant approach for video understanding $[5,21,33,39,46-48,50,51,56]$. This includes the highly successful two-stream networks $[21,39,50]$ and $3 \mathrm{D}$ convolutional networks $[5,33,46-48,51,56]$. In this paper, we use 3D CNNs, but the long-term feature bank can be integrated with other families of video models as well.

Temporal and relationship models include RNNs that model the evolution of video frames [7, 24, 27, 44, 57] and multilayer perceptrons that model ordered frame features [58]. To model finer-grained interactions, a growing line of work leverages pre-computed object proposals [52] or detections $[4,30]$, and models their co-occurrence $[30,43$, 52], temporal order [4], or spatial arrangement [52] within a short clip.

Long-term video understanding with modern CNNs is less explored, in part due to GPU memory constraints. One strategy to overcome these constraints is to use precomputed features without end-to-end training [25, 31, 45, 57]. These methods do not optimize features for a target task, and thus are likely suboptimal. Another strategy is to use aggressive subsampling [50,58] or large striding [8]. TSN [50] samples 3-7 frames per video. ST-ResNet [8] uses a temporal stride of 15 . To our knowledge, our approach is the first that enjoys the best of three worlds: end-to-end learning for strong short-term features with dense sampling and decoupled, flexible long-term modeling.

Spatio-temporal action localization is an active research area $[12,14,17,32,40,53]$. Most recent approaches extend object detection frameworks $[10,34]$ to first propose tubelets/boxes in a short clip/frame, and then classify the tubelets/boxes into action classes [14, 17, 20, 32, 36, 36]. The detected tubelets/boxes can then be optionally linked to form full action tubes $[12,17,20,32,36,40]$. In contrast to our method, these methods find actions within each frame or clip independently without exploiting long-term context.

Information 'bank' representations, such as object bank [26], detection bank [1], and memory networks [42] have been used as image-level representations, for video indexing and retrieval, and for modeling information in text corpora. We draw inspiration from these approaches and develop methodologies for detailed video understanding tasks.

\section{Long-Term Feature Bank Models}

For computer vision models to make accurate predictions on long, complex videos they will certainly require the ability to relate what is happening in the present to events that are distant in time. With this motivation in mind, we propose a model with a long-term feature bank to explicitly enable these interactions.

\subsection{Method Overview}

We describe how our method can be used for the task of spatio-temporal action localization, where the goal is to detect all actors in a video and classify their actions. Most state-of-the-art methods [9, 14, 43] combine a 'backbone' 3D CNN (e.g., C3D [46], I3D [5]) with a region-based person detector (e.g., Fast/Faster R-CNN [10,34]). To process a video, it is split into short clips of 2-5 seconds, which are independently forwarded through the $3 \mathrm{D} \mathrm{CNN}$ to compute a feature map, which is then used with region proposals and region of interest (RoI) pooling to compute RoI features for each candidate actor $[9,14]$. This approach, which captures only short-term information, is depicted in Fig. 3 a.

The central idea in our method is to extend this approach with two new concepts: (1) a long-term feature bank that intuitively acts as a 'memory' of what happened during the entire video-we compute this as RoI features from detections at regularly sampled time steps; and (2) a feature bank operator (FBO) that computes interactions between the short-term RoI features (describing what actors are doing now) and the long-term features. The interactions may be computed through an attentional mechanism, such as a non-local block [51], or by feature pooling and concatenation. Our model is summarized in Fig. 3b. We introduce these concepts in detail next.

\subsection{Long-Term Feature Bank}

The goal of the long-term feature bank, $L$, is to provide relevant contextual information to aid recognition at the current time step. For the task of spatio-temporal action localization, we run a person detector over the entire video to generate a set of detections for each frame. In parallel, we run a standard clip-based 3D CNN, such as C3D [46] or I3D [5], over the video at regularly spaced intervals, such as every one second. We then use RoI pooling to extract fea- 


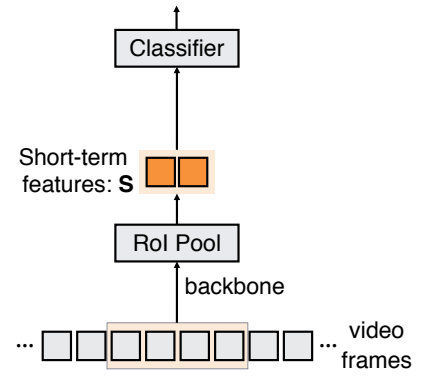

(a) 3D CNN

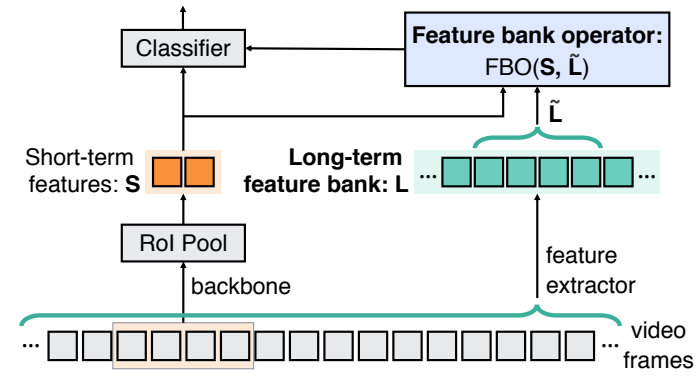

(b) 3D CNN with a Long-Term Feature Bank (Ours)

Figure 3. We contrast our model with standard methods. (a) 3D CNN: vanilla 3D CNNs process a short clip from a video (e.g., 2-5 seconds) and use pooling to obtain a representation of the clip (e.g., [5, 46, 51]). (b) Long-Term Feature Bank (Ours): We extend the vanilla $3 \mathrm{D} C N N$ with a long-term feature bank $L$ and a feature bank operator $\operatorname{FBO}(S, \tilde{L})$ that computes interactions between the short-term and long-term features. Our model is able to integrate information over a long temporal support, lasting minutes or even the whole video.

tures for all person detections at each time-step processed by the $3 \mathrm{D}$ CNN. Formally, $L=\left[L_{0}, L_{1}, \ldots, L_{T-1}\right]$ is a time-indexed list of features for video time steps $0, \ldots, T-$ 1 , where $L_{t} \in \mathbb{R}^{N_{t} \times d}$ is the matrix of $N_{t} d$-dimensional RoI features at time $t$. Intuitively, $L$ provides information about when and what all actors are doing in the whole video and it can be efficiently computed in a single pass over the video by the detector and 3D CNN.

\subsection{Feature Bank Operator}

Our model references information from the long-term features $L$ via a feature bank operator, $\operatorname{FBO}\left(S_{t}, \tilde{L}_{t}\right)$. The feature bank operator accepts inputs $S_{t}$ and $\tilde{L}_{t}$, where $S_{t}$ is the short-term RoI pooled feature and $\tilde{L}_{t}$ is $\left[L_{t-w}, \ldots, L_{t+w}\right]$, a subset of $L$ centered at the current clip at $t$ within 'window' size $2 w+1$, stacked into a matrix $\tilde{L}_{t} \in \mathbb{R}^{N \times d}$, where $N=\sum_{t^{\prime}=t-w}^{t+w} N_{t^{\prime}}$. We treat the window size $2 w+1$ as a hyperparameter that we cross-validate in our experiments. The output is then channel-wise concatenated with $S_{t}$ and used as input into a linear classifier.

Intuitively, the feature bank operator computes an updated version of the pooled short-term features $S_{t}$ by relating them to the long-term features. The implementation of FBO is flexible. Variants of attentional mechanisms are an obvious choice and we will consider multiple instantiations in our experiments.

Batch vs. Casual. Thus far we have assumed a batch setting in which the entire video is available for processing. Our model is also applicable to online, casual settings. In this case, $\tilde{L}_{t}$ contains only past information of window size $2 w+1$; we consider both batch and causal modes of operation in our experiments.

\subsection{Implementation Details}

Backbone. We use a standard 3D CNN architecture from recent video classification work. The model is a ResNet50 [16] that is pre-trained on ImageNet [35] and 'inflated' into a network with $3 \mathrm{D}$ convolutions (over space and time) using the I3D technique [5]. The network structure is modified to include non-local operations [51]. After inflating the network from $2 \mathrm{D}$ to $3 \mathrm{D}$, we pre-train it for video classification on the Kinetics-400 dataset [5]. The model achieves $74.9 \%$ (91.6\%) top-1 (top-5) accuracy on the Kinetics-400 [5] validation set. Finally, we remove the temporal striding for conv ${ }_{1}$ and pool $_{1}$ following [52], and remove the Kinetics-specific classification layers to yield the backbone model. The exact model specification is given in the Appendix. The resulting network accepts an input of shape $32 \times H \times W \times 3$, representing 32 RGB frames with spatial size $H \times W$, and outputs features with shape $16 \times H / 16 \times W / 16 \times 2048$. The same architecture is used to compute short-term features $S$ and long-term features $L$. Parameters are not shared between these two models unless otherwise noted.

RoI Pooling. We first average pool the video backbone features over the time axis. We then use RoIAlign [15] with a spatial output of $7 \times 7$, followed by spatial max pooling, to yield a single 2048 dimensional feature vector for the RoI. This corresponds to using a temporally straight tube [14].

Feature Bank Operator Instantiations. The feature bank operator can be implemented in a variety of ways. We experiment with the following choices; others are possible.

- LFB NL: Our default feature bank operator $\mathrm{FBO}_{\mathrm{NL}}\left(S_{t}, \tilde{L}_{t}\right)$ is an attention operator. Intuitively, we use $S_{t}$ to attend to features in $\tilde{L}_{t}$, and add the attended information back to $S_{t}$ via a shortcut connection. We use a simple implementation in which $\mathrm{FBO}_{\mathrm{NL}}\left(S_{t}, \tilde{L}_{t}\right)$ is a stack of up to three non-local (NL) blocks [51]. We replace the selfattention of the standard non-local block [51] with attention between the local features $S_{t}$ and the long-term feature window $\tilde{L}_{t}$, illustrated in Fig. 4 . In addition, our design uses layer normalization (LN) [3] and dropout [41] to improve regularization. We found these modifications to be important since our target tasks contain relatively few training videos and exhibit overfitting. The stack of modified 


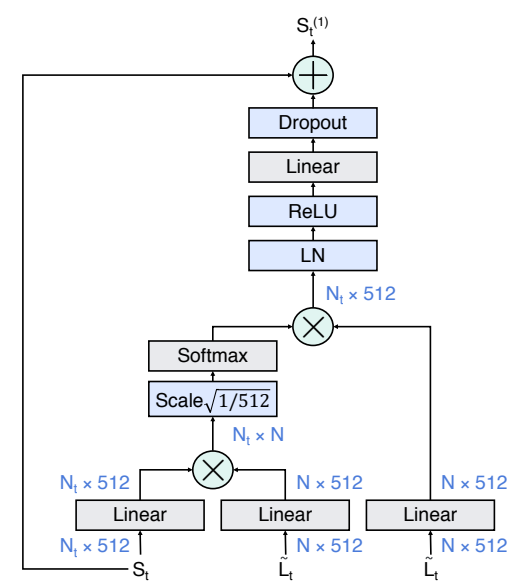

Figure 4. Our modified non-local block design. Here we plot the first layer $S_{t}^{(1)}=\mathrm{NL}_{\theta_{1}}\left(S_{t}, \tilde{L}_{t}\right)$ as an example. ' $\otimes$ ' denotes matrix multiplication, and ' $\oplus$ ' denotes element-wise sum.

non-local blocks, denoted as $\mathrm{NL}^{\prime}$, is iterated as:

$$
\begin{aligned}
& S_{t}^{(1)}=\mathrm{NL}_{\theta_{1}}^{\prime}\left(S_{t}, \tilde{L}_{t}\right), \\
& S_{t}^{(2)}=\mathrm{NL}_{\theta_{2}}^{\prime}\left(S_{t}^{(1)}, \tilde{L}_{t}\right),
\end{aligned}
$$

where $\theta_{\{1,2, \ldots\}}$ are learnable parameters. Similar to Wang et al. [52], we use a linear layer to reduce the $\mathrm{FBO}_{\mathrm{NL}}$ input dimensionality to 512 and apply dropout [41] with rate 0.2 . Thus the input of the final linear classifier is $2048\left(S_{t}\right)$ $+512\left(\mathrm{FBO}_{\mathrm{NL}}\right.$ output $)=2560$ dimensional.

- LFB Avg/Max: This version uses a simpler variant in which $\mathrm{FBO}_{\text {pool }}\left(S_{t}, \tilde{L}_{t}\right)=\operatorname{pool}\left(\tilde{L}_{t}\right)$, where pool can be either average or max pooling. This implementation results in a classifier input that is $2048\left(S_{t}\right)+2048\left(\mathrm{FBO}_{\text {pool }}\right.$ output $)$ $=4096$ dimensional.

Training. Joint, end-to-end training of the entire model (Fig. 3b) is not feasible due to the computational and memory complexity of back-propagating through the long-term feature bank. Instead, we treat the 3D CNN and detector that are used to compute $L$ as fixed components that are trained offline, but still on the target dataset, and not updated subsequently. We have experimented with alternating optimization methods for updating these models, similar to target propagation [23], but found that they did not improve results. Dataset-specific training details are given later.

A Baseline Short-Term Operator. To validate the benefit of incorporating long-term information, we also study a 'degraded' version of our model that does not use a longterm feature bank. Instead, it uses a short-term operator that is identical to $\mathrm{FBO}_{\mathrm{NL}}$, but only references the information from within a clip: $\operatorname{STO}\left(S_{t}\right):=\mathrm{FBO}_{\mathrm{NL}}\left(S_{t}, S_{t}\right)$. STO is conceptually similar to [52] and allows for backpropagation. We observed substantial overfitting with STO and thus applied additional regularization techniques. See the Appendix for details.

\section{Experiments on AVA}

We use the AVA dataset [14] for extensive ablation studies. AVA consists of 235 training videos and 64 validation videos; each video is a 15 minute segment taken from a movie. Frames are labeled sparsely at 1 FPS. The labels are: one bounding box around each person in the frame together with a multi-label annotation specifying which actions the person in the box is engaged in within $\pm 0.5 \mathrm{sec}$ onds of the labeled frame. The action label space is defined as 80 'atomic' actions defined by the dataset authors.

The task in AVA is spatio-temporal action localization: each person appearing in a test video must be detected in each frame and the multi-label actions of the detected person must be predicted correctly. The quality of an algorithm is judged by a mean average precision (mAP) metric that requires at least 50\% intersection over union (IoU) overlap for a detection to be matched to the ground-truth while simultaneously predicting the correct actions.

\subsection{Implementation Details}

Next, we describe the object detector, input sampling, and training and inference details used for AVA.

Person Detector. We use Faster R-CNN [34] with a ResNeXt-101-FPN [28, 55] backbone for person detection. The model is pre-trained on ImageNet [35] and COCO keypoints [29], and then fine-tuned on AVA bounding boxes; see the Appendix for training details. The final model obtains 93.9 AP@50 on the AVA validation set.

Temporal Sampling. Both short- and long-term features are extracted by 3D CNNs that use 32 input frames sampled with a temporal stride of 2 spanning 63 frames ( $\sim 2$ seconds in 30 FPS video). Long-term features are computed at one clip per second over the whole video, with a 3D CNN model (Fig. 3a) fine-tuned on AVA.

Training. We train our models using synchronous SGD with a minibatch size of 16 clips on 8 GPUs (i.e., 2 clips per GPU), with batch normalization [18] layers frozen. We train all models for 140k iterations, with a learning rate of 0.04 , which is decreased by a factor of 10 at iteration $100 \mathrm{k}$ and $120 \mathrm{k}$. We use a weight decay of $10^{-6}$ and momentum of 0.9. For data augmentation, we perform random flipping, random scaling such that the short side $\in[256,320]$ pixels, and random cropping of size $224 \times 224$. We use both ground-truth boxes and predicted boxes with scores at least 0.9 for training. This accounts for the discrepancy between ground-truth-box distribution and predicted-box distribution, and we found it beneficial. We assign labels of a ground-truth box to a predicted box if they overlap with IoU at least 0.9. A predicted box might have no labels assigned. Since the number of long-term features $N$ differs from clip to clip, we pad zero-vectors for clips with fewer long-term features to simplify minibatch training. 


\begin{tabular}{lccccccc} 
Support (sec.) & 2 & 3 & 5 & 10 & 15 & 30 & 60 \\
\hline 3D CNN & 22.1 & 22.2 & 22.3 & 20.0 & 19.7 & 17.5 & 15.7 \\
STO & 23.2 & 23.6 & 23.3 & 21.5 & 20.9 & 18.5 & 16.9 \\
LFB (causal) & - & 24.0 & 24.3 & 24.6 & 24.8 & 24.6 & 24.2 \\
LFB (batch) & - & 24.2 & 24.7 & 25.2 & $\underline{25.3}$ & $\underline{25.3}$ & $\mathbf{2 5 . 5}$
\end{tabular}

(a) Temporal support (mAP in \%)

\begin{tabular}{|c|c|c|c|c|c|}
\hline & $\mathrm{mAP}$ & & params & FLOPs & $\mathrm{mAP}$ \\
\hline $1 \mathrm{~L}$ & 25.1 & 3D CNN & $1 \times$ & $1 \times$ & 22.1 \\
\hline 2L (default) & $\underline{25.5}$ & $3 \mathrm{D} \mathrm{CNN} \times 2$ & $2 \times$ & $2 \times$ & 22.9 \\
\hline 2L w/o scale & 25.2 & STO & $1.00 \times$ & $1.12 \times$ & 23.2 \\
\hline 2L w/o LN & 23.9 & $\mathrm{STO} \times 2$ & $2.00 \times$ & $2.24 \times$ & 24.1 \\
\hline 2L w/o dropout & 25.4 & LFB (2L) & $2.00 \times$ & $2.12 \times$ & $\underline{25.5}$ \\
\hline 2L (dot product) & $\underline{25.5}$ & LFB (3L) & $2.00 \times$ & $2.15 \times$ & 25.8 \\
\hline 2L (concat) & 25.3 & & & & \\
\hline $3 \mathrm{~L}$ & 25.8 & & & & \\
\hline
\end{tabular}

(e) LFB NL design

\begin{tabular}{lr} 
& mAP \\
\hline K400 feat. & 19.7 \\
AVA feat. & $\underline{24.3}$ \\
LFB & $\mathbf{2 5 . 5}$
\end{tabular}

(b) Feature decoupling

\begin{tabular}{lr} 
& mAP \\
\hline Avg pool & 23.1 \\
Max pool & $\underline{23.2}$ \\
NL & $\mathbf{2 5 . 5}$
\end{tabular}

(c) LFB operator

\begin{tabular}{lr} 
& mAP \\
\hline Global pool & 24.9 \\
$2 \times 2$ Grid & $\underline{25.1}$ \\
$4 \times 4$ Grid & $\underline{25.1}$ \\
Detection & $\mathbf{2 5 . 5}$
\end{tabular}

(d) LFB spatial design

\begin{tabular}{|c|c|c|c|c|c|}
\hline & mAP & model & flow & val & test \\
\hline R50-I3D-NL & & $\overline{\text { AVA [14] }}$ & $\checkmark$ & 15.6 & - \\
\hline center-crop (default) & 25.8 & ACRN [43] & $\checkmark$ & 17.4 & - \\
\hline R101-I3D-NL & & RTPR [24] & $\checkmark$ & 22.3 & - \\
\hline center-crop (default) & 26.8 & 9-model ens. [19] & $\checkmark$ & 25.6 & 21.1 \\
\hline 3-crop & 27.1 & R50-I3D-NL [19] & & 19.3 & - \\
\hline 3-crop+flips & 27.4 & RTPR [24] & & 20.5 & - \\
\hline \multirow[t]{3}{*}{ 3-crop+flips+3-scale } & 27.7 & Girdhar et al. [9] & & 21.9 & 21.0 \\
\hline & & LFB (R50) & & $\underline{25.8}$ & 24.8 \\
\hline & & LFB (best R101) & & $\overline{27.7}$ & $\overline{27.2}$ \\
\hline
\end{tabular}

(g) Backbone \& testing

Table 1. AVA ablations and test results. STO: 3D CNN with a non-local (NL) short-term operator; LFB: 3D CNN with a long-term feature bank; the LFB operator is a two-layer (2L) NL block by default. We perform ablations on the AVA spatio-temporal action localization. The results validate that longer-term information is beneficial, that the improvement is larger than what would be observed by ensembling, and demonstrate various design choices. Finally, we show state-of-the-art results on the AVA test set.

Inference. At test time we use detections with scores $\geq$ 0.85 . All models rescale the short side to 256 pixels and use a single center crop of $256 \times 256$. For both training and inference, if a box crosses the cropping boundary, we pool the region within the cropped clip. In the rare case where a box falls out of the cropped region, RoIAlign [15] pools the feature at the boundary.

\subsection{Ablation Experiments}

Temporal Support. We first analyze the impact of increasing temporal support on models both with and without LFB. For models without LFB, we evaluate a vanilla 3D CNN (Fig. 3a) and a 3D CNN extended with STO (denoted 'STO' in tables). To increase their temporal support, we increase the temporal stride but fix the number of input frames so that the model covers a longer temporal range while still being feasible to train. To increase the temporal support of LFB models, we increase the 'window size' $2 w+1$ of $\tilde{L}_{t}$.

Table 1a compares model performance. Increasing temporal support through striding in fact hurts the performance of both '3D CNN' and 'STO'. Temporal convolution might not be suitable for long-term patterns, since long-term patterns are more diverse, and include challenging scene cuts. On the other hand, increasing temporal support by adding an LFB steadily improves performance, leading to a large gain over the original '3D CNN' $(22.1 \rightarrow \mathbf{2 5 . 5})$. The online (causal) setting shows a similar trend.

Overall we observe strong improvements given by longrange context even though AVA actions are designed to be 'atomic' and localized within \pm 0.5 seconds. For the rest of the ablation study, we focus on the batch setting and use a window size of 60 seconds due to the strong performance.
Feature Decoupling. In Table $1 \mathrm{~b}$, we compare our decoupled feature approach with prior long-term modeling strategies where a single, pre-computed feature type is used (e.g. $[25,31,45,57])$. To do this, we use the same 3D CNN for both short-term and long-term feature bank computation and keep it fixed during training; only the parameters of the FBO and classifier are updated. We consider two choices: a Kinetics-400 [5] pre-trained 3D CNN ('K400 feat.') and a 3D CNN that is fine-tuned on AVA ('AVA feat.'). Our decoupled approach, which updates the short-term 3D CNN based on the long-term context, works significantly better.

FBO Function Design. We next compare different FBO function designs in Table 1c. We see that a non-local function significantly outperforms pooling on AVA. This is not surprising, since videos in AVA (and videos in general) are multi-actor, multi-action, and can exhibit complex interactions possibly across a long range of time. We expect a more complex function class is required for reasoning through the complex scenes. Nonetheless, pooling still offers a clear improvement. This again confirms the importance of longterm context in video understanding.

FBO Input Design. What spatial granularity is required for complex video understanding? In Table 1d, we compare constructing long-term features using detected objects ('Detection'), regular grids ('Grid'), and non-spatial features ('Global pool'). In the 'Grid' experiments, we divide the feature map into a $k \times k$ grid, for $k=2,4$, and average the features within each bin to obtain a localized representation without an object detector (similar to ACRN [43]).

Table 1d shows that the actor-level features ('Detection') works better than coarser regular-grid or non-spatial fea- 


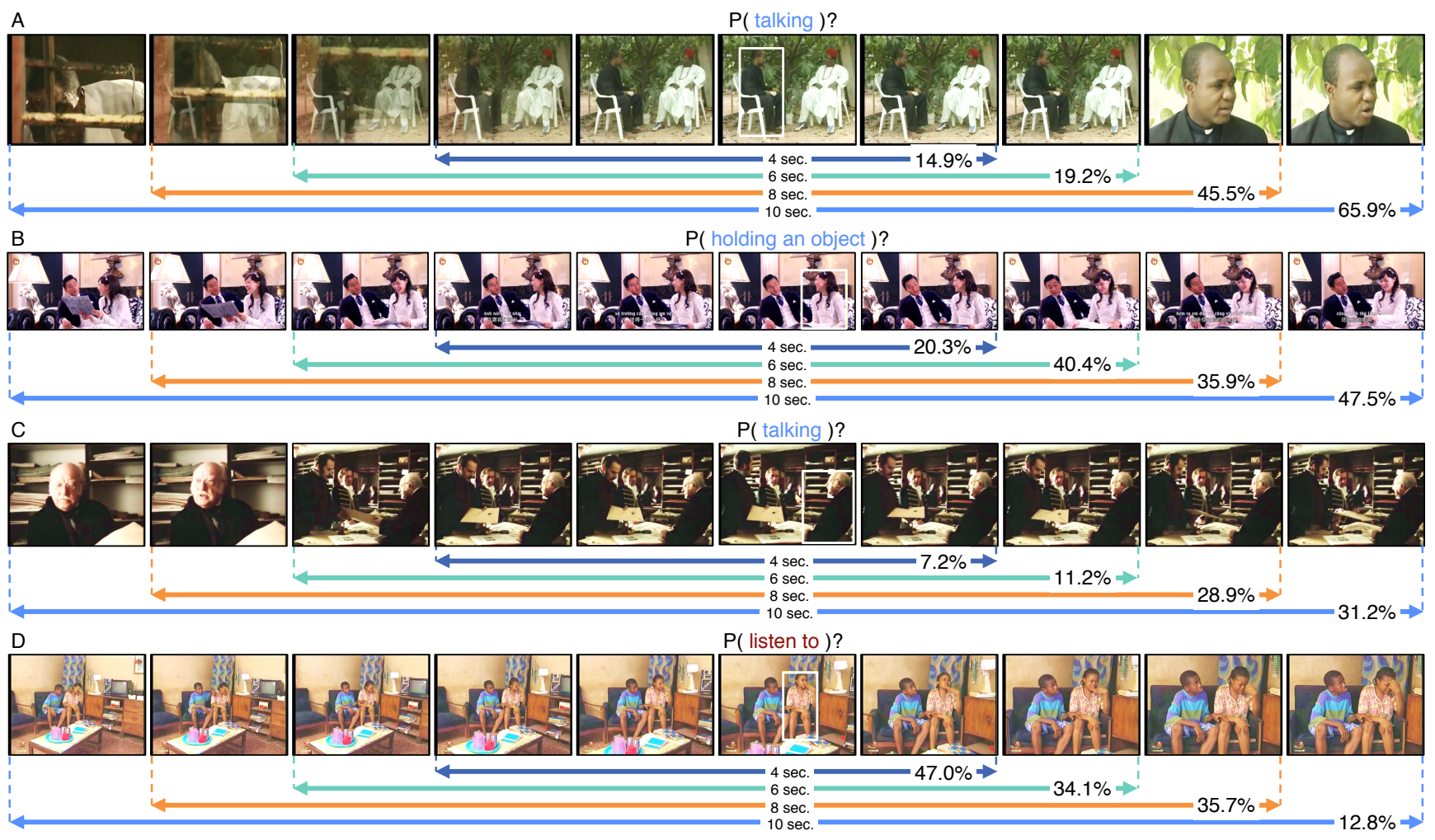

Figure 5. Example Predictions. We compare predictions made by models using LFB of different window sizes. Through LFB, the model is able to exploit information distant in time, e.g., the zoomed-in frames in example A and C, to improve predictions. We encourage readers to zoom in for details. (Blue: correct labels. Red: incorrect labels. Best viewed on screen.)

tures. We believe this suggests a promising future research direction that moves from global pooling to a more detailed modeling of objects/actors in video.

Non-Local Block Design. Next, we ablate our NL block design. In Table 1e, we see that adding a second layer of NL blocks leads to improved performance. In addition, scaling [49], layer normalization [3], and dropout [41] all contribute to good performance. Layer normalization [3], which is part of our modified NL design, is particularly important. As found in [51], the default embedded Gaussian variant performs similarly to dot product and concatenation. (Later we found that adding a third layer of NL blocks further improves accuracy, but otherwise use two by default.)

Model Complexity. Our approach uses two instances of the backbone model: one to compute the long-term features and another to compute the short-term features. It thus uses around $2 \times$ more parameters and computation than our baselines. What if we simply use $2 \times$ more computation on baselines through an ensemble? We find that both '3D CNN' or 'STO' are not able to obtain a similar gain when ensembled; the LFB model works significantly better (Table 1f).

Example Predictions. We qualitatively present a few examples illustrating the impact of LFB in Fig. 5. Specifically, we compare predictions made by models with LFB of different window sizes, from 4 seconds to 10 seconds. We see that when observing only short-term information, the model is confused and not able to make accurate predictions in these cases. When observing more context, e.g., zoomedin frames (example A, C) or frames that give clearer cue (example B, D), an LFB model is able to leverage the information and improve predictions.

Backbone and Testing. So far for simplicity, we have used a relatively small backbone of R50-I3D-NL and performed center-crop testing. In Table $1 \mathrm{~g}$, we show that with an R101-I3D-NL backbone, LFB (3L) achieves $26.8 \mathrm{mAP}$, and with standard testing techniques, $27.7 \mathrm{mAP}$. We use short side $\in\{224,256,320\}$ pixels for 3 -scale testing.

Comparison to Prior Work. Finally we compare with other state-of-the-art methods (Table 1h). For fair comparison, we follow Girdhar et al. [9] and train on both the training and validation set for test set evaluation. ${ }^{2}$ For this model we use a $1.5 \times$ longer schedule due to the larger data size.

Our model, using only RGB frames, significantly outperforms all prior work including strong competition winners that use optical flow and large ensembles. Our single model outperforms the best previous single-model entry by Girdhar et al. [9] with a margin of 5.8 and 6.2 points mAP on validation and test set respectively.

\footnotetext{
${ }^{2}$ Test set performance evaluated by ActivityNet server.
} 


\section{Experiments on EPIC-Kitchens}

The long-term feature bank is a generalizable and flexible concept. We illustrate this point on two tasks in the EPIC-Kitchens dataset [6] for which we store different types of information in the feature bank.

The EPIC-Kitchens dataset consists of videos of daily activities (mostly cooking) recorded in participants' native kitchen environments. Segments of each video are annotated with one verb (e.g., 'squeeze') and one noun (e.g., 'lemon'). The task is to predict the verb, noun, and the combination (termed action [6]) in each segment. Performance is measured by top- 1 and top- 5 accuracy.

The dataset consists of 39,594 segments in 432 videos. Test set annotations are not released; for validation we split the original training set into a new training/validation split following Baradel et al. [4]. We train independent models to recognize verbs and nouns, and combine their predictions for actions. For actions, we additionally use a prior based on the training class frequencies of verbs and nouns; see the Appendix for details.

\subsection{Implementation Details}

Long-Term Feature Banks. Recognizing which object a person is interacting with (the noun task) from a short segment is challenging, because the object is often occluded, blurred, or can even be out of the scene. Our LFB is wellsuited for addressing these issues, as the long-term supporting information can help resolve ambiguities. For example, if we know that the person took a lemon from the refrigerator 30 seconds ago, then cutting a lemon should be more likely. Based on this motivation, we construct an LFB that contains object-centric features. Specifically, we use Faster R-CNN to detect objects and extract object features using RoIAlign from the detector's feature maps (see the Appendix for details of our detector).

On the other hand, for recognizing verbs we use a video model to construct an LFB that captures motion patterns. Specifically, we use our baseline 3D CNN fine-tuned on EPIC-Kitchens verbs to extract clip-level features every 1 second of video. Our default setting uses a window size of 40 seconds for the verb model, and 12 seconds for the noun model, chosen on the validation set.

Adaptation to Segment-Level Tasks. To adapt our model for segment-level predictions, we replace the RoI pooling by global average pooling, resulting in $S \in \mathbb{R}^{1 \times 2048}$. For the STO baseline, we use a slightly modified formulation: $\operatorname{STO}\left(S_{t}^{\prime}\right):=\mathrm{FBO}_{\mathrm{NL}}\left(S_{t}, S_{t}^{\prime}\right)$, where $S_{t}^{\prime}$ contains 16 spatially pooled features, each at a temporal location, and $S_{t}$ is $S_{t}^{\prime}$ pooled over the time axis. STO learns to interact with information at different time steps within the short clip. Training and inference procedures are analogous to our experiments on AVA; see the Appendix for details.

\begin{tabular}{lcccccc} 
& \multicolumn{2}{c}{ Verbs } & \multicolumn{2}{c}{ Nouns } & \multicolumn{2}{c}{ Actions } \\
\cline { 2 - 7 } & top-1 & top-5 & top-1 & top-5 & top-1 & top-5 \\
\hline Validation & & & & & & \\
Baradel [4] & 40.9 & - & - & - & - & - \\
3D CNN & 49.8 & 80.6 & 26.1 & 51.3 & 19.0 & 37.8 \\
3D CNN ens. & 50.7 & $\underline{81.2}$ & 27.8 & 52.8 & 20.0 & 39.0 \\
STO & 51.0 & 80.8 & 26.6 & 51.5 & 19.5 & 38.3 \\
STO ens. & 51.9 & $\underline{81.2}$ & 27.8 & 52.5 & 20.5 & 39.4 \\
LFB NL & 51.7 & $\underline{81.2}$ & $\underline{29.2}$ & 55.3 & $\underline{21.4}$ & 40.2 \\
LFB Avg & $\mathbf{5 3 . 0}$ & $\mathbf{8 2 . 3}$ & 29.1 & $\underline{55.4}$ & 21.2 & 40.8 \\
LFB Max & $\underline{52.6}$ & $\underline{81.2}$ & $\mathbf{3 1 . 8}$ & $\mathbf{5 6 . 8}$ & $\mathbf{2 2 . 8}$ & $\mathbf{4 1 . 1}$ \\
\multicolumn{1}{c}{$\Delta$} & +3.2 & +1.7 & +5.7 & +5.5 & +3.8 & +3.3 \\
\hline Test s1 (seen) & & & & & & \\
TSN RGB [6] & 45.7 & 85.6 & 36.8 & 64.2 & 19.9 & 41.9 \\
TSN Flow [6] & 42.8 & 79.5 & 17.4 & 39.4 & 9.0 & 21.9 \\
TSN Fusion [6] & 48.2 & 84.1 & 36.7 & 62.3 & 20.5 & 39.8 \\
LFB Max & $\mathbf{6 0 . 0}$ & $\mathbf{8 8 . 4}$ & $\mathbf{4 5 . 0}$ & $\mathbf{7 1 . 8}$ & $\mathbf{3 2 . 7}$ & $\mathbf{5 5 . 3}$ \\
\hline Test s2 (unseen) & & & & & & \\
TSN RGB [6] & 34.9 & 74.6 & 21.8 & 45.3 & 10.1 & 25.3 \\
TSN Flow [6] & 40.1 & 73.4 & 14.5 & 33.8 & 6.7 & 18.6 \\
TSN Fusion [6] & 39.4 & 74.3 & 22.7 & 45.7 & 10.9 & 25.3 \\
LFB Max & $\mathbf{5 0 . 9}$ & $\mathbf{7 7 . 6}$ & $\mathbf{3 1 . 5}$ & $\mathbf{5 7 . 8}$ & $\mathbf{2 1 . 2}$ & $\mathbf{3 9 . 4}$ \\
& & & & & &
\end{tabular}

Table 2. EPIC-Kitchens validation and test server results. Augmenting 3D CNN with LFB leads to significant improvement.

\subsection{Quantitative Evaluation}

We now quantitatively evaluate our LFB models. Table 2 shows that augmenting a 3D CNN with LFB significantly boosts the performance for all three tasks. Using object features for the noun model is particularly effective, leading to $5.7 \%(26.1 \rightarrow 31.8)$ absolute improvement over our strong baseline model. On verb recognition, LFB with 3D CNN features results in $3.2 \%(49.8 \rightarrow \mathbf{5 3 . 0})$ improvement and outperforms previous state-of-the-art by Baradel et al. [4] by $12.1 \%(40.9 \rightarrow \mathbf{5 3 . 0})$.

We also observe that $\mathrm{FBO}_{\mathrm{Max}}$ and $\mathrm{FBO}_{\text {Avg }}$ outperform $\mathrm{FBO}_{\mathrm{NL}}$ on EPIC-Kitchens. We conjecture that this is due to the simpler setting: each video has only one person, doing one thing at a time, without the complicated person-person interactions of AVA. Thus a simpler function suffices.

On the test set, our method outperforms prior work by a large margin on both the 'seen kitchens (s1)' and 'unseen kitchens (s2)' settings. Our LFB model outperforms the Two-Stream [39] TSN [50] baseline by 50\% relatively for $\mathrm{s} 1$, and almost doubles the performance for $\mathrm{s} 2$, in terms of top-1 action accuracy.

\section{Experiments on Charades}

Finally we evaluate our approach on the Charades dataset [38]. The Charades dataset contains 9,848 videos with an average length of 30 seconds. In each video, a person can perform one or more actions. The task is to recognize all the actions in the video without localization. 


\begin{tabular}{lrr} 
iterations / lr / wd & $50 \mathrm{k} / 0.0025 / 1 \mathrm{e}-4[52]$ & $24 \mathrm{k} / 0.02 / 1.25 \mathrm{e}-5$ \\
\hline 3D CNN & 33.8 & $\mathbf{3 8 . 3}$ \\
STO & 37.8 & $\mathbf{3 9 . 6}$
\end{tabular}

Table 3. Training schedule on Charades. Our $2 \times$ shorter schedule works significantly better than the schedule used in STRG [52].

\begin{tabular}{lllcc} 
& backbone & modality & $\begin{array}{c}\text { train / } \\
\text { val }\end{array}$ & $\begin{array}{c}\text { trainval } \\
\text { / test }\end{array}$ \\
\hline 2-Strm. [39] (from [37]) & VGG16 & RGB+Flow & 18.6 & - \\
Asyn-TF [37] & VGG16 & RGB+Flow & 22.4 & - \\
CoViAR [54] & R50 & Compressed & 21.9 & - \\
MultiScale TRN [58] & Inception & RGB & 25.2 & - \\
I3D [5] & Inception-I3D & RGB & 32.9 & 34.4 \\
I3D [5] (from [51]) & R101-I3D & RGB & 35.5 & 37.2 \\
I3D-NL [51] (from [52]) & R50-I3D-NL & RGB & 33.5 & - \\
I3D-NL [51] & R101-I3D-NL & RGB & 37.5 & 39.5 \\
STRG [52] & R50-I3D-NL & RGB & 37.5 & - \\
STRG [52] & R101-I3D-NL & RGB & 39.7 & - \\
\hline 3D CNN & R50-I3D-NL & RGB & 38.3 & - \\
3D CNN ens. & R50-I3D-NL & RGB & 39.5 & - \\
STO & R50-I3D-NL & RGB & 39.6 & - \\
STO ens. & R50-I3D-NL & RGB & 40.0 & - \\
LFB Avg & R50-I3D-NL & RGB & 38.4 & - \\
LFB Max & R50-I3D-NL & RGB & 38.6 & - \\
LFB NL & R50-I3D-NL & RGB & $\mathbf{4 0 . 3}$ & - \\
\hline 3D CNN & R101-I3D-NL & RGB & 40.3 & 40.8 \\
3D CNN ens. & R101-I3D-NL & RGB & 41.7 & - \\
STO & R101-I3D-NL & RGB & 41.0 & - \\
STO ens. & R101-I3D-NL & RGB & 42.3 & - \\
LFB Avg & R101-I3D-NL & RGB & 40.8 & - \\
LFB Max & R101-I3D-NL & RGB & 40.9 & - \\
LFB NL & R101-I3D-NL & RGB & $\mathbf{4 2 . 5}$ & $\mathbf{4 3 . 4}$ \\
Tabl 4. Acto & RG &
\end{tabular}

Table 4. Action recognition accuracy on Charades. (mAP in \%)

\subsection{Implementation Details}

We use the RGB frames at 24 FPS provided by the dataset authors. We sample training and testing clips (32 frames) with a temporal stride of 4 following STRG [52], resulting in input clips spanning 125 frames ( $\sim 5.2$ seconds). The LFB is sampled at 2 clips per second. We found a postactivation version of $\mathrm{NL}^{\prime}$ to work better on Charades, so we adopt it in the following experiments. Details and full results of both variants are in the Appendix. Other details are identical to the verb model for EPIC-Kitchens.

Training and Inference. We train 3D CNN models for $24 \mathrm{k}$ iterations with a learning rate of 0.02 and weight decay of $1.25 \mathrm{e}-5$. Note that these hyperparameters are different from STRG [52], which uses longer schedule (50k iterations), smaller learning rate $(0.0025)$, and larger weight decay (1e-4). ${ }^{3}$ Table 3 compares the two settings, and we see that surprisingly our $2 \times$ shorter schedule works significantly better. With the new schedule, a simple NL model without proposals (STO) works as well as the full STRG

\footnotetext{
${ }^{3}$ The original STRG [52] uses a batch size of 8. For clear comparison, we use the same batch size as ours (16), but adjust the learning rate and schedule according to 'Linear Scaling Rule' [13]. We verified that the accuracy matches that of the original 4-GPU training.
}

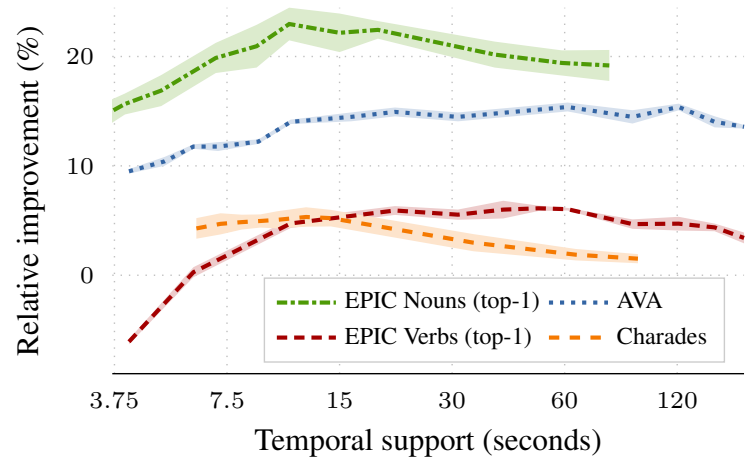

Figure 6. Relative improvement of LFB models with different window sizes over vanilla 3D CNN.

method (37.5\% mAP) [52]. We observe that the benefit of using the short-term operator becomes smaller when using a stronger baseline. In all following experiments we use our $24 \mathrm{k}$ schedule as default, and use a 2-stage training approach similar to STRG [52] for training LFB models; see the Appendix for details. At test time, we sample 10 clips per video, and combine the predictions using max pooling following prior work [51,52]. We use (left, center, right) 3-crop testing following Wang et al. [51].

\subsection{Quantitative Evaluation}

For Charades, we experiment with both ResNet-50-I3DNL and ResNet-101-I3D-NL [5, 16, 51] backbones for a consistent comparison to prior work. Table 4 shows that LFB models again consistently outperform all models without LFB, including prior state-of-the-art on both validation and test sets. The improvement on Charades is not as large as other datasets, in part due to the coarser prediction task (video-level).

\section{Discussion}

Fig. 6 shows the relative gain of using LFB of different window sizes. ${ }^{4}$ We see that different datasets exhibit different characteristics. The movie dataset, AVA, benefits from very long context lasting $2+$ minutes. To recognize cooking activities (EPIC-Kitchens), context spanning from 15 to 60 seconds is useful. Charades videos are much shorter $(\sim 30$ seconds), but still, extending the temporal support to $10+$ seconds is beneficial. We conjecture that more challenging datasets in the future may benefit even more.

In conclusion, we propose a Long-Term Feature Bank that provides long-term supportive information to video models. We show that enabling video models with access to long-term information, through an LFB, leads to a large performance gain and yields state-of-the-art results on challenging datasets like AVA, EPIC-Kitchens, and Charades.

\footnotetext{
${ }^{4}$ For each dataset, we use its best-performing FBO. Standard error is calculated based on 5 runs. The temporal support here considers the support of each clip used for computing $L$, so Charades's support starts at a higher value due to its larger $3 \mathrm{D}$ CNN clip size ( $~ 5.2$ seconds).
} 


\section{Appendix A. Backbone Architecture}

We use ResNet-50 I3D [14, 16] with non-local blocks [51] as the 'backbone' of our model. Following Wang et al. [52], the network only downsamples the temporal dimension by a factor of two. Table 5 presents the exact specification.

\begin{tabular}{|c|c|c|c|}
\hline Stage & \multicolumn{2}{|c|}{ Specification } & Output size \\
\hline$\overline{\operatorname{conv}_{1}}$ & \multicolumn{2}{|c|}{$5 \times 7 \times 7,64$, stride $1,2,2$} & $32 \times 112 \times 112$ \\
\hline $\operatorname{pool}_{1}$ & \multicolumn{2}{|c|}{$1 \times 3 \times 3$ max, stride $1,2,2$} & $32 \times 56 \times 56$ \\
\hline $\operatorname{res}_{2}$ & $\begin{array}{l}3 \times 1 \times 1,64 \\
1 \times 3 \times 3,64 \\
1 \times 1 \times 1,256\end{array}$ & $\times 3$ & $32 \times 56 \times 56$ \\
\hline $\mathrm{pool}_{2}$ & \multicolumn{2}{|c|}{$2 \times 1 \times 1$ max, stride $2,1,1$} & $16 \times 56 \times 56$ \\
\hline $\operatorname{res}_{3}$ & $\begin{array}{c}3(1) \times 1 \times 1,128 \\
1 \quad \times 3 \times 3,128 \\
1 \quad \times 1 \times 1,512\end{array}$ & $\times 4$, NL: 1,3 & $16 \times 28 \times 28$ \\
\hline res $_{4}$ & $\begin{array}{l}3(1) \times 1 \times 1,256 \\
1 \quad \times 3 \times 3,256 \\
1 \quad \times 1 \times 1,1024\end{array}$ & $\times 6$, NL: $1,3,5$ & $16 \times 14 \times 14$ \\
\hline $\operatorname{res}_{5}$ & $\begin{array}{l}3(1) \times 1 \times 1,512 \\
1 \quad \times 3 \times 3,512 \\
1 \quad \times 1 \times 1,2048\end{array}$ & $\times 3$ & $16 \times 14 \times 14$ \\
\hline
\end{tabular}

Table 5. ResNet-50 NL-I3D [14, 16, 51] backbone used in this paper. Here we assume input size $32 \times 224 \times 224$ (frames $\times$ width $\times$ height). 'NL: $i_{0}, i_{1}, \ldots$ ' in stage 'res ${ }_{j}$ ' denotes additional non-local blocks [51] after block $i_{0}, i_{1}, \ldots$ of res $_{j}$. $3(1) \times 1 \times 1$ denotes that we either use a $3 \times 1 \times 1$ or a $1 \times 1 \times 1$ convolution. Specifically, we use $3 \times 1 \times 1$ for block 0,2 of res 3 , block $0,2,4$ of res $_{4}$, and block 1 of res 5 , and use $1 \times 1 \times 1$ for the rest.

\section{Appendix B. AVA Person Detector}

We use Faster R-CNN [34] with a ResNeXt-101FPN $[28,55]$ backbone for person detection. The model is pre-trained on ImageNet for classification, and on COCO for keypoint detection. The model obtains 56.9 box AP and 67.0 keypoint AP on COCO keypoints. Model parameters are available in Detectron Model Zoo [11]. We fine-tune the model on AVA bounding boxes from the training videos for $130 \mathrm{k}$ iterations with an initial learning rate of 0.005 , which is decreased by a factor of 10 at iteration $100 \mathrm{k}$ and $120 \mathrm{k}$. To improve generalization, we train with random scale jittering (from 512 to 800 pixels). The final model obtains 93.9 AP@50 on the AVA validation set.

\section{Appendix C. LFB vs. Improving Backbones}

A large body of recent research focuses on improving 3D $\mathrm{CNN}$ architectures, i.e., improving modeling of short-term patterns. This paper, on the other hand, aims at improving the modeling of long-term patterns. How do these two directions impact video understanding differently?

We plot the per-class impact of LFB in Fig. 7, the perclass impact of improving backbone in Fig. 8, and compare them in Fig. 9. The error bars are plus/minus one standard error around the mean, computed from 5 runs. We see that they lead to improvement in different action classes. Using LFB leads to improvement in many interactive actions, such as 'play musical instrument' or 'sing to', while improving backbone leads to improvement in more standalone actions, such as 'hand shake' or 'climb'. This suggests that improving long-term modeling (through LFB) and short-term modeling (through improving backbone) are complementary; we believe both are important for future video understanding research.

\section{Appendix D. AVA STO Regularization}

To address the overfitting issue of STO on AVA, we experimented with a number of regularization techniques, as summarized in Table 6. We found that dropout [41] was insufficient to regularize an STO, but injecting 'distractors', i.e., randomly sampled features, into the features being attended during training was very effective. We report STO results with 'distractor' training for AVA unless otherwise stated. For STO on other datasets, we did not observe obvious overfitting.

\begin{tabular}{ccccc} 
3D CNN & STO & $\begin{array}{c}\text { STO } \\
+0.5 \text { dropout }\end{array}$ & $\begin{array}{c}\text { STO } \\
+0.8 \text { dropout }\end{array}$ & $\begin{array}{c}\text { STO } \\
+ \text { distractors }\end{array}$ \\
\hline 22.1 & 20.2 & 20.1 & 20.9 & $\mathbf{2 3 . 2}$
\end{tabular}

Table 6. STO with different regularization techniques on AVA (mAP in \%).

\section{Appendix E. Training Schedule for EPIC- Kitchens}

We train the verb models for $36 \mathrm{k}$ iterations with $10^{-5}$ weight decay and a learning rate of 0.0003 , which is then decreased by 10 times at iteration $28 \mathrm{k}$ and $32 \mathrm{k}$. For the noun models, we train for 50k iterations with weight decay $10^{-6}$ and a learning rate of 0.001 , which is decreased by 10 times at iteration $40 \mathrm{k}$ and $45 \mathrm{k}$.

\section{Appendix F. EPIC-Kitchens Inference}

We sample training clips such that the center of the clip falls within a training segment. For testing, we sample one center clip per segment, resize such that the short side is 256 pixels, and use a single center crop of $256 \times 256$. We compute the probability of an action as the product of the softmax scores, weighted by a prior $\mu$, i.e.

$P($ action $=(v, n)) \propto \mu(v, n) P(\operatorname{verb}=v) P($ noun $=n)$,

where $\mu$ is a prior estimated as the count of $(v, n)$ pair divided by count of $n$ in training annotations. 


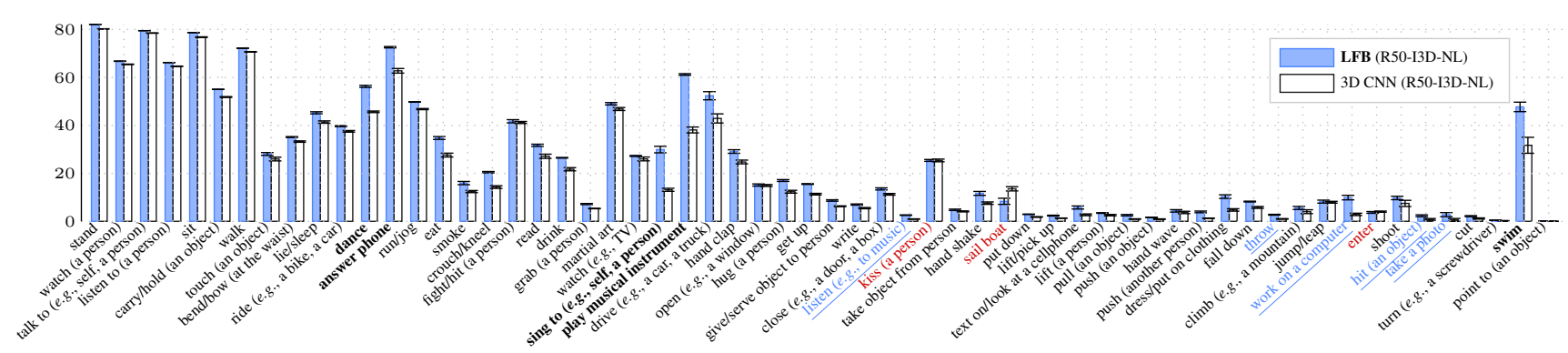

Figure 7. Impact of LFB. We compare per-class AP of 3D CNN (22.1 mAP) and LFB model (25.5 mAP) on AVA. LFB leads to larger improvement on interactive actions, e.g., 'sing to', 'play musical instrument', or 'work on a computer'. (Bold: 5 classes with the largest absolute gain. Blue: 5 classes with the largest relative gain. Red: classes with decreased performance. Classes are sorted by frequency. )

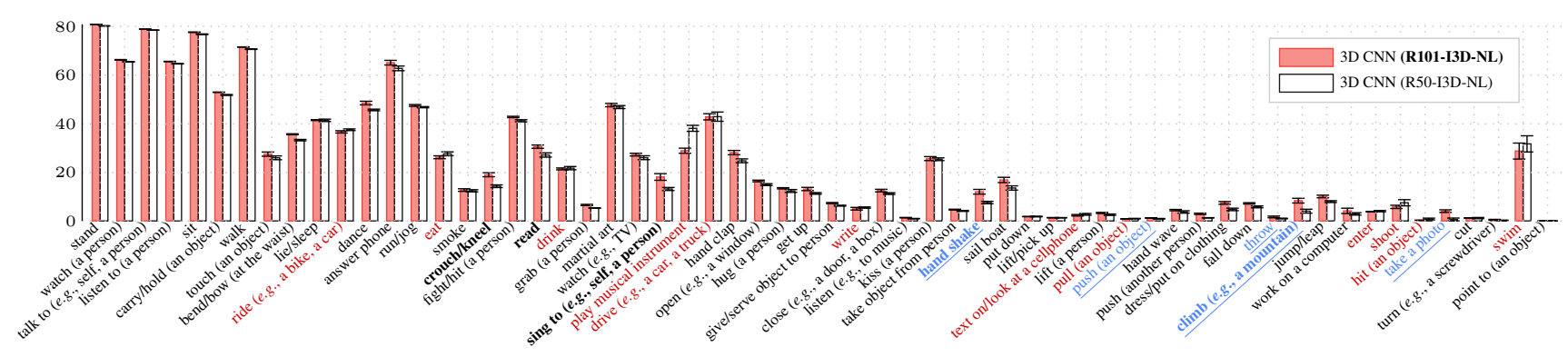

Figure 8. Impact of improving backbone. We compare per-class AP of 3D CNN with the default backbone (R50-I3D-NL; 22.1 mAP) and a stronger backbone (R101-I3D-NL; $23.0 \mathrm{mAP}$ ) on AVA. Improving backbone leads to larger improvement in standalone actions, such as 'crouch/kneel', 'read', or 'hand shake'. (Bold: 5 classes with the largest absolute gain. Blue: 5 classes with the largest relative gain. Red: classes with decreased performance. )

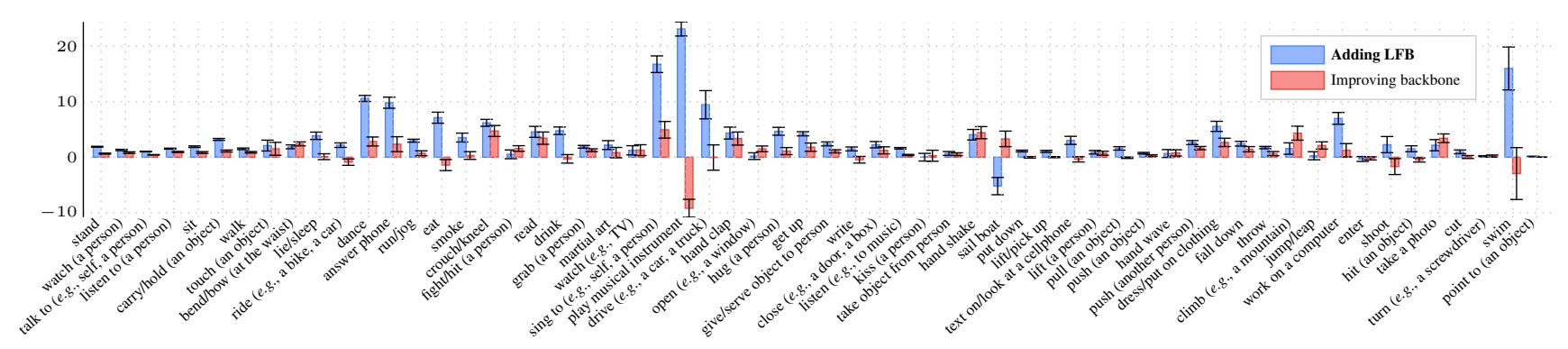

Figure 9. Adding LFB vs. improving backbone. We compare the absolute improvement (in AP) brought by LFB and the improvement brought by improving backbone. We see that they lead to improvement in different action classes. This suggests that improving long-term modeling (through LFB) and short-term modeling (through improving backbone) are complementary; we believe both are important for future video understanding research.

\section{Appendix G. Object Detector for LFB of EPIC-Kitchens Noun Model}

We use Faster R-CNN [34] with ResNeXt-101-FPN [28, 55] backbone for the object detector. The detector is pretrained on Visual Genome [22] with 1600 class labels defined in Anderson et al. [2]. We fine-tune this model on the 'new' training split (defined in Baradel et al. [4]) of EPIC-Kitchens for 90k iterations, with random scale jittering (from 512 to 800 pixels). We use an initial learning rate of 0.005 , which is decreased by a factor of 10 at itera- tion $60 \mathrm{k}$ and $80 \mathrm{k}$. The final model achieves $2.4 \mathrm{AP}$ on the 'new' validation split. The AP is low because with the new train/val split, most of the classes are unseen during training. In addition, most of the classes have zero instance in the new, smaller validation set, and we calculate the average precision of those classes as 0 . This is also not comparable to the performance reported in [6], where the model is trained on the full training set, and evaluated on the unreleased test sets. 


\section{Appendix H. Charades Training Schedule}

We train the models to predict the 'clip-level' labels, i.e., the union of the frame labels that fall into the clip's temporal range. We train the baseline 3D CNNs with the default $24 \mathrm{k}$ schedule with learning rate 0.02 , which is decreased by a factor of 10 at iteration 20k. To train LFB models, we use a 2-stage approach following STRG [52]. We first train the model without the FBO using the $24 \mathrm{k}$ schedule, and then add FBO, freeze backbone, and train for half of the schedule (12k iterations, so $36 \mathrm{k}$ in total). This schedule helps prevent overfitting that was observed when training directly with FBO in one stage. For training STO, we observed worse performance with this 2-stage approach, so we report the STO performance using the default $24 \mathrm{k}$ schedule.

\section{Appendix I. Charades NL Block Details}

\begin{tabular}{l|cc|cc} 
& \multicolumn{2}{|c|}{ R50-I3D-NL } & \multicolumn{2}{c}{ R101-I3D-NL } \\
& pre-act & post-act & pre-act & post-act \\
\hline STO & 39.0 & 39.6 & 40.5 & 41.0 \\
LFB NL & 39.5 & $\mathbf{4 0 . 3}$ & 41.5 & $\mathbf{4 2 . 5}$ \\
\hline 3D CNN & \multicolumn{2}{|c|}{38.3} & \multicolumn{2}{|c}{40.3}
\end{tabular}

Table 7. Pre-activation vs. post-activation $\mathrm{NL}^{\prime}$ on Charades.

We experimented with two variants of $\mathrm{NL}^{\prime}$ design: a pre-activation (the default variant described in paper), and a post-activation variant, where we move ReLU after the skip connection, and move the layer normalization after the output linear layer. For both variants, LFB consistently outperforms STO and baseline 3D CNN (Table 7). We choose post-activation as default for Charades due to the stronger performance. For AVA and EPIC-Kitchens, we did not observe any noticeable difference between the two variants.

\section{References}

[1] T. Althoff, H. O. Song, and T. Darrell. Detection bank: an object detection based video representation for multimedia event recognition. In International Conference on Multimedia, 2012. 2

[2] P. Anderson, X. He, C. Buehler, D. Teney, M. Johnson, S. Gould, and L. Zhang. Bottom-up and top-down attention for image captioning and visual question answering. In CVPR, 2018. 10

[3] J. L. Ba, J. R. Kiros, and G. E. Hinton. Layer normalization. arXiv preprint arXiv:1607.06450, 2016. 3, 6

[4] F. Baradel, N. Neverova, C. Wolf, J. Mille, and G. Mori. Object level visual reasoning in videos. In $E C C V, 2018$. 2, 7, 10

[5] J. Carreira and A. Zisserman. Quo vadis, action recognition? a new model and the kinetics dataset. In $C V P R, 2017.1,2$, $3,5,8$

[6] D. Damen, H. Doughty, G. M. Farinella, S. Fidler, A. Furnari, E. Kazakos, D. Moltisanti, J. Munro, T. Perrett,
W. Price, et al. Scaling egocentric vision: The EPIC-kitchens dataset. In ECCV, 2018. 1, 7, 10

[7] J. Donahue, L. Anne Hendricks, S. Guadarrama, M. Rohrbach, S. Venugopalan, K. Saenko, and T. Darrell. Long-term recurrent convolutional networks for visual recognition and description. In $C V P R, 2015.2$

[8] C. Feichtenhofer, A. Pinz, and R. Wildes. Spatiotemporal residual networks for video action recognition. In NIPS, 2016. 2

[9] R. Girdhar, J. Carreira, C. Doersch, and A. Zisserman. A better baseline for AVA. arXiv preprint arXiv:1807.10066, 2018. 2, 5, 6

[10] R. Girshick. Fast R-CNN. In ICCV, 2015. 2

[11] R. Girshick, I. Radosavovic, G. Gkioxari, P. Dollár, and K. He. Detectron, 2018. 9

[12] G. Gkioxari and J. Malik. Finding action tubes. In $C V P R$, 2015. 2

[13] P. Goyal, P. Dollár, R. Girshick, P. Noordhuis, L. Wesolowski, A. Kyrola, A. Tulloch, Y. Jia, and K. He. Accurate, large minibatch sgd: Training imagenet in 1 hour. arXiv preprint arXiv:1706.02677, 2017. 8

[14] C. Gu, C. Sun, D. A. Ross, C. Vondrick, C. Pantofaru, Y. Li, S. Vijayanarasimhan, G. Toderici, S. Ricco, R. Sukthankar, et al. AVA: A video dataset of spatio-temporally localized atomic visual actions. In CVPR, 2018. 1, 2, 3, 4, 5, 9

[15] K. He, G. Gkioxari, P. Dollár, and R. Girshick. Mask RCNN. In ICCV, 2017. 3, 5

[16] K. He, X. Zhang, S. Ren, and J. Sun. Deep residual learning for image recognition. In $C V P R, 2016.3,8,9$

[17] R. Hou, C. Chen, and M. Shah. Tube convolutional neural network (T-CNN) for action detection in videos. In ICCV, 2017. 2

[18] S. Ioffe and C. Szegedy. Batch normalization: Accelerating deep network training by reducing internal covariate shift. In ICML, 2015. 4

[19] J. Jiang, Y. Cao, L. Song, S. Z. Y. Li, Z. Xu, Q. Wu, C. Gan, C. Zhang, and G. Yu. Human centric spatio-temporal action localization. In ActivityNet workshop, CVPR, 2018. 5

[20] V. Kalogeiton, P. Weinzaepfel, V. Ferrari, and C. Schmid. Action tubelet detector for spatio-temporal action localization. In $I C C V, 2017.2$

[21] A. Karpathy, G. Toderici, S. Shetty, T. Leung, R. Sukthankar, and L. Fei-Fei. Large-scale video classification with convolutional neural networks. In CVPR, 2014. 2

[22] R. Krishna, Y. Zhu, O. Groth, J. Johnson, K. Hata, J. Kravitz, S. Chen, Y. Kalantidis, L.-J. Li, D. A. Shamma, et al. Visual genome: Connecting language and vision using crowdsourced dense image annotations. IJCV, 2017. 10

[23] Y. LeCun. Learning process in an asymmetric threshold network. In Disordered systems and biological organization. 1986. 4

[24] D. Li, Z. Qiu, Q. Dai, T. Yao, and T. Mei. Recurrent tubelet proposal and recognition networks for action detection. In ECCV, 2018. 2, 5

[25] F. Li, C. Gan, X. Liu, Y. Bian, X. Long, Y. Li, Z. Li, J. Zhou, and S. Wen. Temporal modeling approaches for large-scale youtube-8m video understanding. arXiv preprint arXiv:1707.04555, 2017. 1, 2, 5 
[26] L.-J. Li, H. Su, L. Fei-Fei, and E. P. Xing. Object bank: A high-level image representation for scene classification \& semantic feature sparsification. In NIPS, 2010. 2

[27] Z. Li, K. Gavrilyuk, E. Gavves, M. Jain, and C. G. Snoek. Videolstm convolves, attends and flows for action recognition. Computer Vision and Image Understanding, 2018. 2

[28] T.-Y. Lin, P. Dollár, R. B. Girshick, K. He, B. Hariharan, and S. J. Belongie. Feature pyramid networks for object detection. In CVPR, 2017. 4, 9, 10

[29] T.-Y. Lin, M. Maire, S. Belongie, J. Hays, P. Perona, D. Ramanan, P. Dollár, and C. L. Zitnick. Microsoft coco: Common objects in context. In ECCV, 2014. 4

[30] C.-Y. Ma, A. Kadav, I. Melvin, Z. Kira, G. AlRegib, and H. P. Graf. Attend and interact: Higher-order object interactions for video understanding. In $C V P R, 2018.2$

[31] A. Miech, I. Laptev, and J. Sivic. Learnable pooling with context gating for video classification. arXiv preprint arXiv:1706.06905, 2017. 1, 2, 5

[32] X. Peng and C. Schmid. Multi-region two-stream r-cnn for action detection. In ECCV, 2016. 2

[33] Z. Qiu, T. Yao, and T. Mei. Learning spatio-temporal representation with pseudo-3d residual networks. In ICCV, 2017. 1,2

[34] S. Ren, K. He, R. Girshick, and J. Sun. Faster R-CNN: Towards real-time object detection with region proposal networks. In NIPS, 2015. 2, 4, 9, 10

[35] O. Russakovsky, J. Deng, H. Su, J. Krause, S. Satheesh, S. Ma, Z. Huang, A. Karpathy, A. Khosla, M. Bernstein, A. C. Berg, and L. Fei-Fei. ImageNet Large Scale Visual Recognition Challenge. IJCV, 2015. 3, 4

[36] S. Saha, G. Singh, and F. Cuzzolin. AMTnet: Action-microtube regression by end-to-end trainable deep architecture. In ICCV, 2017. 2

[37] G. A. Sigurdsson, S. K. Divvala, A. Farhadi, and A. Gupta. Asynchronous temporal fields for action recognition. In CVPR, 2017. 8

[38] G. A. Sigurdsson, G. Varol, X. Wang, A. Farhadi, I. Laptev, and A. Gupta. Hollywood in homes: Crowdsourcing data collection for activity understanding. In ECCV, 2016. 1, 7

[39] K. Simonyan and A. Zisserman. Two-stream convolutional networks for action recognition in videos. In NIPS, 2014. 2, 7,8

[40] G. Singh, S. Saha, M. Sapienza, P. H. Torr, and F. Cuzzolin. Online real-time multiple spatiotemporal action localisation and prediction. In $I C C V, 2017.2$

[41] N. Srivastava, G. Hinton, A. Krizhevsky, I. Sutskever, and R. Salakhutdinov. Dropout: a simple way to prevent neural networks from overfitting. JMLR, 2014. 3, 4, 6, 9

[42] S. Sukhbaatar, A. Szlam, J. Weston, and R. Fergus. End-toend memory networks. In NIPS, 2015. 2

[43] C. Sun, A. Shrivastava, C. Vondrick, K. Murphy, R. Sukthankar, and C. Schmid. Actor-centric relation network. In ECCV, 2018. 2, 5

[44] L. Sun, K. Jia, K. Chen, D.-Y. Yeung, B. E. Shi, and S. Savarese. Lattice long short-term memory for human action recognition. In $I C C V, 2017.2$
[45] Y. Tang, X. Zhang, J. Wang, S. Chen, L. Ma, and Y.-G. Jiang. Non-local netvlad encoding for video classification. arXiv preprint arXiv:1810.00207, 2018. 1, 2, 5

[46] D. Tran, L. Bourdev, R. Fergus, L. Torresani, and M. Paluri. Learning spatiotemporal features with $3 \mathrm{~d}$ convolutional networks. In ICCV, 2015. 1, 2, 3

[47] D. Tran, J. Ray, Z. Shou, S.-F. Chang, and M. Paluri. Convnet architecture search for spatiotemporal feature learning. arXiv preprint arXiv:1708.05038, 2017. 1, 2

[48] G. Varol, I. Laptev, and C. Schmid. Long-term temporal convolutions for action recognition. PAMI, 2018. 1, 2

[49] A. Vaswani, N. Shazeer, N. Parmar, J. Uszkoreit, L. Jones, A. N. Gomez, Ł. Kaiser, and I. Polosukhin. Attention is all you need. In NIPS, 2017. 6

[50] L. Wang, Y. Xiong, Z. Wang, Y. Qiao, D. Lin, X. Tang, and L. Van Gool. Temporal segment networks: Towards good practices for deep action recognition. In ECCV, 2016. 2, 7

[51] X. Wang, R. Girshick, A. Gupta, and K. He. Non-local neural networks. In $C V P R, 2018.1,2,3,6,8,9$

[52] X. Wang and A. Gupta. Videos as space-time region graphs. In $E C C V, 2018.1,2,3,4,8,9,11$

[53] P. Weinzaepfel, Z. Harchaoui, and C. Schmid. Learning to track for spatio-temporal action localization. In ICCV, 2015. 2

[54] C.-Y. Wu, M. Zaheer, H. Hu, R. Manmatha, A. J. Smola, and P. Krähenbühl. Compressed video action recognition. In CVPR, 2018. 8

[55] S. Xie, R. Girshick, P. Dollár, Z. Tu, and K. He. Aggregated residual transformations for deep neural networks. In CVPR, 2017. 4, 9, 10

[56] S. Xie, C. Sun, J. Huang, Z. Tu, and K. Murphy. Rethinking spatiotemporal feature learning for video understanding. In ECCV, 2018. 1, 2

[57] J. Yue-Hei Ng, M. Hausknecht, S. Vijayanarasimhan, O. Vinyals, R. Monga, and G. Toderici. Beyond short snippets: Deep networks for video classification. In CVPR, 2015. $1,2,5$

[58] B. Zhou, A. Andonian, A. Oliva, and A. Torralba. Temporal relational reasoning in videos. In $E C C V, 2018.2,8$ 